\title{
Gustaaf VERSWIJVER, Les Kararaô du Brésil central
}

\section{Stéphanie Tselouiko}

\section{(2) OpenEdition \\ Journals}

\section{Édition électronique}

URL : https://journals.openedition.org/jsa/19729

DOI : 10.4000/jsa. 19729

ISSN : 1957-7842

\section{Éditeur}

Société des américanistes

\section{Édition imprimée}

Date de publication : 30 septembre 2021

Pagination : 249-255

ISSN : 0037-9174

Référence électronique

Stéphanie Tselouiko, "Gustaaf verswiJver, Les Kararaô du Brésil central », Journal de la Société des américanistes [En ligne], 107-1 | 2021, mis en ligne le 30 septembre 2021, consulté le 04 septembre 2022. URL : http://journals.openedition.org/jsa/19729; DOI : https://doi.org/10.4000/jsa.19729

Ce document a été généré automatiquement le 4 septembre 2022.

Tous droits réservés 


\title{
Gustaaf VERSWIJVER, Les Kararaô du Brésil central
}

\author{
Stéphanie Tselouiko
}

\section{RÉFÉRENCE}

Gustaaf VERSWIJVER, Les Kararaô du Brésil central, Fondation culturelle musée Barbier-

Mueller, Genève/Ides et Calendes, Lausanne, 2020, 208 p., bibliogr., append., ill. (en noir et en coul.), photos (en noir et en coul.), cartes, tabl., graph.

1 Les Kayapó du Brésil (ou plus exactement les Mẽbêngôkre), appartenant au tronc linguistique Gé, comptent actuellement un peu plus de 9000 individus divisés en six grands groupes: les Gorotire, les Kubenkranken, les Kokraimoro, les Mekranoti, les Xikrin et les Kararaô. Si les cinq premiers ont été amplement étudiés depuis les années 1960 par de nombreux ethnologues, les Kararaô sont quant à eux demeurés dans l'ombre. La sortie du dernier ouvrage de Gustaaf Verswijver, Les Kararaô du Brésil central, contribuera à remédier à cette lacune mais aussi à mieux la comprendre.

2 Comment, en effet, expliquer ce manque d'intérêt suscité par ce peuple chez les ethnologues? L'auteur avance que l'absence, dans l'unique village Kararaô connu, d'activités rituelles et de maison des hommes (caractéristiques de la société mẽbêngôkre considérées comme essentielles) explique que la plupart des chercheurs ${ }^{1}$ ait préféré s'intéresser aux communautés plus grandes, présentant une vie politique et rituelle plus riche $^{2}$. L'objectif de cet ouvrage est donc de mettre en lumière l'histoire singulière de la constitution du groupe Kararaô, qui se caractérise par une suite ininterrompue de scissions et de fusions. L'auteur met en évidence le rôle catalyseur qu'ont eu les relations avec les colons brésiliens et les missionnaires sur la dynamique de leur population de même que sur l'accroissement des conflits et la montée en puissance de la violence entre les groupes divisés. Ces relations ont profondément influencé le comportement de ces Amérindiens, caractérisé par le pillage institutionnalisé et l'enlèvement de femmes et 
d'enfants. Elles ont aussi motivé leur "volonté obsessionnelle d'isolement » (p. 164) et abouti à une configuration sans pareil dans l'histoire des Mẽbêngôkre.

Cette enquête ne part pas de nulle part, puisque Gustaaf Verswijver mène des recherches chez les Mekranoti depuis le milieu des années 1970. Il est d'ailleurs sans doute le spécialiste des Mẽbêngôkre le plus considéré par ses pairs, non seulement pour sa maîtrise de leur langue, mais aussi pour sa contribution fondamentale à la compréhension de leur organisation socio-politique ainsi que pour la documentation de leur histoire au cours du $\mathrm{xx}^{\mathrm{e}}$ siècle. Reposant sur ses recherches antérieures, mais aussi à l'issue de trois missions ethnographiques réalisées entre 2015 et 2017 et d'une importante recherche en archives, l'auteur se propose ici de compléter le puzzle socio-historique complexe des Mẽbêngôkre. Sans compter d'innombrables discussions informelles, ce travail s'appuie sur 79 entretiens enregistrés auprès des Kararaô, des Mekranoti, mais aussi de Brésiliens qui ont été en contact plus ou moins direct avec les Kararaô et qui ont pu apporter des informations précieuses sur des moments clés de leur histoire. Le travail d'archives est considérable et puise dans 37 sources provenant aussi bien d'articles de presse parus entre 1937 et 1996 que dans les archives du Service de protection des Indiens (SPI) des années 1950 et 1960.

Le lecteur ne doit cependant pas s'attendre à trouver dans cet ouvrage une théorisation anthropologique poussée. C'est un récit ethno-historique que l'auteur s'est attaché à réaliser, un « voyage à travers le temps et l'espace » (p. 9). Et il faut avouer que ce voyage ne se fait pas sans peine et sans une certaine désorientation parfois, tant le parcours est complexe et les évènements relatés nombreux. Le texte est d'ailleurs complété de près de 200 notes qui apportent des précisions très importantes, mais rendent parfois la lecture un peu moins fluide. Cependant l'auteur parvient malgré tout à ne jamais perdre son lecteur grâce à un récit vivant et passionnant. Les anecdotes qu'il livre à travers l'histoire de vie de certains individus confèrent à cette recherche ethno-historique une dimension affective. Les cartes, les tableaux synthétiques et les diagrammes fournissent des repères précieux au fur et à mesure de la lecture : treize cartes, dont deux provenant d'archives, retracent les itinéraires et les emplacements des différentes factions au cours de l'histoire jusqu'à présent, y compris les emplacements supposés de groupes actuellement toujours isolés. Quant aux photos, très nombreuses (et parfois douloureuses), elles contribuent à se rapprocher intimement des individus et de leur vie quotidienne. Elles sont aussi choisies et placées avec attention pour faire apparaître au lecteur la complexité des rapports entre les différents groupes mẽbêngôkre. À cet égard, la première photo (p. 2) qui représente un homme mekranoti devenu chef de l'actuel village des Kararaô de l'Iriri, laisse présager de façon un peu énigmatique la circulation des personnes et du pouvoir entre les différents groupes dont les relations ont oscillé entre guerre et paix. Sur les 170 photos, une centaine est issue d'archives, souvent de photographes inconnus, certaines remontent aux années 1930, c'est-à-dire lors des tout premiers contacts, et la plus ancienne date de 1909.

5 Chacun, connaisseur ou non, aura plaisir à suivre le voyage auquel Verswijver nous convie. Dans le premier chapitre, il fournit les informations indispensables pour comprendre l'organisation socio-politique des Mẽbêngôkre, leur rapport à l'espace, leur vie rituelle et leurs activités productives. Si l'auteur a su restituer de manière claire et concise les données produites par les nombreux chercheurs ayant étudié les Mẽbêngôkre à différentes périodes, on peut toutefois regretter quelques raccourcis, notamment en ce qui concerne le rapport à l'environnement et la tendance à le présenter dans un schéma 
dichotomique nature/culture. Mais comme les spécialistes des Mẽbêngôkre le savent, il y a des tâches beaucoup plus aisées que celle de résumer en quelques pages la vaste littérature produite sur ce peuple depuis les années 1970. Dans ce chapitre introductif, l'auteur esquisse également l'histoire des Mẽbêngôkre au cours du xvIII siècle. Celle-ci, marquée par de nombreuses scissions, a abouti à la configuration de plusieurs groupes dont les Xikrin, les Ira'amranh (aujourd'hui disparus) et les Krinkati (« ceux du grand village »), qui constituaient le groupe mẽbêngôkre le plus important resté uni dans un village, appelé Pukatôti ${ }^{3}$. C'est là que commence l'histoire des Kararaô. À la fin du $\mathrm{XIX}^{\mathrm{e}}$ siècle, Pukatôti rassemblaient six sociétés des hommes réparties en deux maisons (Ouest et Est) ${ }^{4}$. Les deux premières décennies du $\mathrm{Xx}^{\mathrm{e}}$ siècle verront naître les tensions issues de la rencontre entre les colons brésiliens et les Krinkati, moment où les conditions de dépendance de plus en plus forte de ces derniers aux produits manufacturés occidentaux, et en particulier les armes à feu, vont commencer à s'implanter.

6 Dans le second chapitre l'auteur expose les facteurs qui ont conduit à la genèse des Kararaô. D'après les estimations de l'auteur, qui s'appuie sur celles de Darell Posey (1979), Pukatôti devait compter entre 2500 et 4000 individus ce qui en faisait l'un des plus grands, si ce n'est le plus grand, village autochtone de toute l'Amazonie. Toutefois Verswijver explique qu'il était rare que toute la population y soit réunie en même temps; des tensions y naissaient souvent et certains groupes partaient temporairement pour trouver la paix loin du village. Il ne s'agissait pas tant de défection, que de protestation. Parmi les Mẽbêngôkre, l'expression publique du désaccord politique est évitée le plus possible. L'éloignement apparaît alors non seulement comme une manière d'alléger les tensions mais aussi de protester tout en maintenant la cohésion du groupe tant qu'il reste du loyalisme, et ce jusqu'au moment d'atteindre un point de non-retour (Bamberger 1979). Ces mobilités étaient aussi motivées par la quête des biens occidentaux tant désirés. À l'occasion de ces pillages, des femmes et des enfants étaient aussi capturés. Ces informations confirment l'idée selon laquelle les dynamiques des grands groupes amazoniens étaient davantage déterminées par des facteurs sociologiques, politiques et économiques, que par des facteurs écologiques comme la perte de fertilité des sols ou la rareté du gibier.

7 Les Kararaô sont issus de l'une des sociétés des hommes (du même nom) de la maison Ouest de Pukatôti. À travers l'onomastique des noms des différentes sociétés des hommes, l'auteur explique, d'un point de vue non seulement sémantique mais aussi historique, la différenciation des Kararaô vis-à-vis des autres sociétés masculines de ce village. Selon lui, les Kararaô se démarquaient de celles-là par leur caractère particulièrement belliqueux qui leur valut cette appellation (kararaô = onomatopée présente dans leur cri de guerre). Un tel nom est d'ailleurs singulier car généralement, les noms des sociétés des hommes renvoient à des caractéristiques plutôt moqueuses ou ironiques. Or il se trouve que la société des hommes de Pukatôti la plus active dans le pillage des colonies brésiliennes était justement celle des Kararaô. En outre, Kôkôiaumti, le leader des Kararaô à cette époque était un homme particulièrement agressif, y compris envers sa propre communauté. Il était considéré comme un mauvais leader du point de vue des membres des autres sociétés des hommes et était devenu une source de division inévitable. À la suite de nombreux conflits internes, les Kararaô se sont donc constitués en tant que groupe autonome en se faisant chasser définitivement du village de Pukatôti, lequel a continué par la suite d'être la scène de conflits internes et a vu sa population se 
scinder en trois autres groupes. Se sont divisés tout d'abord, les Menokanê et les Kubenkranken, tous deux issus de la maison des hommes Ouest (comme les Kararaô). Les Kubenkranken sont restés à Pukatôti tandis que les Menokanê ont tenté de rejoindre les Mekranoti partis plusieurs années plus tôt. Quant aux Djudjetukti, société des hommes issus de la maison des hommes Est, ils ont quitté également le village ancestral avec les membres des autres sociétés de cette maison et se sont rapprochés pacifiquement d'une colonie brésilienne dans le but d'obtenir protection et surtout des armes pour se défendre des autres groupes mẽbêngôkre, devenus ennemis.

$\mathrm{Au}$ cours de leurs pérégrinations, la question se posait parfois aux groupes ayant fait sécession de prendre une direction donnée pour rejoindre une autre faction ou bien d'aller à l'opposé pour au contraire l'éviter. Ces choix pouvaient être la source de divergences internes qui donnaient lieu à des scissions supplémentaires constituant de nouvelles factions qui tentaient parfois de se retrouver plus tard. Le troisième chapitre retrace comment cette dynamique a marqué les premières années de l'histoire des Kararaô après la scission, en particulier en relation à l'évolution des Djudjetukti qui, eux aussi, se sont divisés en plusieurs groupes à la suite de désaccords concernant le choix de se rapprocher pacifiquement des Brésiliens ou non. Ceux qui y étaient favorables ont dû fuir les représailles de leurs opposants. Lors de cette fuite, ces dissidents ont rencontré les autres factions Kararaô qui s'étaient réunies et auxquelles ils se sont alliés malgré leurs anciens différends. Cet épisode est capital pour saisir l'aspect exceptionnel de la constitution du groupe Kararaô puisqu'il n'existe dans l'histoire des Mẽbêngôkre aucun autre groupe fondé par l'association de sociétés masculines issues de deux maisons des hommes "opposées». La réunion de ces groupes a permis de fonder un grand village Kararaô, mais celui-ci n'a pas duré longtemps. Subissant l'assaut de membres du groupe Djudjetukti particulièrement belliqueux et vindicatifs suite au désaccord qui les avaient séparés, ils ont été massacrés. Les survivants se sont dispersés à nouveaux en trois factions qui connaitront chacune une destinée différente, mais tout aussi tragique.

Le chapitre quatre permet au lecteur de suivre de manière simultanée ces trois factions. Le lecteur apprend ainsi les causes de leurs divisions et de leurs réunifications, qui rendent compte de l'histoire complexe des configurations socio-spatiales des Kararaô jusqu'à leur situation présente. Ces factions ont toutes subi des massacres qui ont eu raison de leur volonté d'isolement. Épuisées et affamées par les guerres et les déplacements, elles ont finalement cherché à établir des relations pacifiques avec les colons non indigènes. Mais ces relations ont toutes occasionné de graves épidémies, qui ont presque exterminé certains groupes. Une seule de ces trois factions parviendra à reconstituer un village : les Kararaô de l'Iriri (un affluent du Xingu); les survivants des deux autres, en revanche, se répartiront au sein de villages mẽbêngôkre voisins, et en particulier chez les Xikrin de la terre indigène Trincheira Bacajá.

On retiendra de l'histoire des Kararaô la peur qui a guidé leurs parcours et motivé leur recherche d'isolement. Attaqués à de nombreuses reprises par les Mekranoti, les Krinkati, puis les Djudjetukti et les Kubenkranken, ils ont été pourchassés, voire massacrés par les Brésiliens, et enfin accablés par les maladies. Leur isolement dans la fuite les a empêchés de s'établir et de retrouver l'équilibre démographique suffisant pour constituer une communauté proprement dite. Cette situation fut d'autant plus dramatique pour eux qu'ils ont été contraints d'abandonner leurs activités rituelles et leurs institutions politiques. Ils sont donc parvenus à survivre physiquement et surtout socialement, mais au prix d'un renoncement qui apparait presque inimaginable tant les activités rituelles 
et les institutions politiques irriguent des aspects essentiels de la socialité mẽbêngôkre. L'exemple des Kararaô invite donc les anthropologues à adopter un nouveau regard sur la résilience et la manière de se réinventer non seulement des groupes mẽbêngôkre, mais également de toutes les populations amérindiennes en fonction de leur histoire. On apprend également que quelques factions kararaô demeureraient toujours isolées à l'heure actuelle, malgré un contexte où rares sont les espaces amazoniens inexplorés et non appropriés. Non seulement on peut s'en étonner, mais aussi se demander comment ils sont parvenus à se maintenir en isolement volontaire sur le long terme (plusieurs décennies maintenant).

11 Gustaaf Verswijver rapporte que la collecte des informations nécessaire à la rédaction de cet ouvrage ne s'est pas faite sans peine lors de ses dernières enquêtes de terrain, car rares sont les actuels descendants qui connaissent l'histoire de leurs aïeux. En 2015, les derniers gardiens mẽbêngôkre de cette histoire se comptaient seulement au nombre de dix. À la sortie de l'ouvrage, trois d'entre eux étaient décédés. En cette période particulièrement troublée par la nouvelle vague épidémique et l'hostilité du gouvernement de Bolsonaro envers les populations indigènes, on peut considérer que l'auteur est parvenu in extremis à répondre à l'objectif de son éditeur, la Fondation culturelle Barbier Mueller qui vise à faire connaître par ses publications des groupes autochtones et leur histoire " pendant qu'il reste des hommes ayant conservé la mémoire vivante de leur peuple ${ }^{5}$ ». C'est avec impatience que nous attendrons maintenant la sortie de son prochain ouvrage où il promet de distiller une analyse anthropologique plus détaillée de ces mêmes matériaux.

\section{BIBLIOGRAPHIE}

\section{BAMBERGER Joan}

1979 « Exit and voice in Central Brazil: the politics of flight in Kayapó society », in David Maybury-Lewis (dir.), Dialectical Societies. The Gê and Bororo of Central Brazil, Harvard University Press, Cambridge, p. 130-146.

POSEY Darell A.

1979 « Pyka-tô-ti. Kayapó mostra aldeia de origem », Revista da atualidade indígena, 3 (15),

p. 50-57.

\section{TURNER Terence}

1965 Social Structure and Political Organization among the Northern Kayapo, thèse de doctorat en anthropologie sociale et ethnologie, Harvard University, Cambridge (MA).

VERSWIJVER Gustaaf

1985 Considerations on Mekrãgnoti Varfare, thèse de doctorat en sciences sociales, université de Gand.

VERSWIJVER Gustaaf

1992 The Club-Fighters of the Amazon. Warfare among the Kayapo Indians of Central Brazil, Rijksuniversiteit te Gent (Werken uitgegeven door de faculteit van de letteren en wijsbegeerte, 179), Gand. 
VIDAL Lux

1990 [1988] "The Kararaô of the Iriri River », in Leinad Ayer de O. Santos et Lúcia M. M. de Andrade (dir.), Hydroelectric Dams on Brazil's Xingu River and Indigenous Peoples, Cultural Survival (Report, 30), Cambridge (MA), p. 153-156.

\section{NOTES}

1. Hormis quelques rares anthropologues, dont l'auteur lui-même (Verswijver 1985, 1992), Terence Turner (1965) et Lux Vidal (1990 [1988]) qui fournissent quelques données sur l'histoire des Kararaô.

2. Un recensement de 2011 dénombre 54 Kararaô, contre plusieurs centaines de personnes dans les autres groupes. Mais en réalité, cette enquête nous montre que les Kararaô sont plus nombreux qu'on ne pouvait le penser.

3. Les Krinkati de Pukatôti, qui n'existent plus aujourd'hui en tant que tels, ne doivent pas être confondus avec l'actuel sous-groupe timbira du même nom (Krîkati) dont la langue est également de la famille linguistique gé.

4. Dans la société mẽbêngôkre, il n'y a pas de chef qui coordonne à lui tout seul l'ensemble du village, mais il existe des groupes politiques formés par les hommes adultes du village appelés « sociétés des hommes » qui se répartissent en deux maisons, Est et Ouest. Chaque société des hommes possède un, voire plusieurs leaders qui coordonnent les activités rituelles et collectives. L'intégration des jeunes hommes au sein de ces sociétés se fait par le biais de liens amicaux.

5. https://fondation-culturelle-barbier-mueller.org/publications/, consulté le $20 / 07 / 2021$.

\section{AUTEURS}

\section{STÉPHANIE TSELOUIKO}

Laboratoire d'anthropologie sociale 